Relations industrielles

Industrial Relations

\title{
L'Église catholique et le problème social
}

\section{Marcel Clément}

Volume 4, numéro 7, mars 1949

URI : https://id.erudit.org/iderudit/1023501ar

DOI : https://doi.org/10.7202/1023501ar

Aller au sommaire du numéro

Éditeur(s)

Département des relations industrielles de l'Université Laval

ISSN

0034-379X (imprimé)

1703-8138 (numérique)

Découvrir la revue

Citer cet article

Clément, M. (1949). L'Église catholique et le problème social. Relations

industrielles / Industrial Relations, 4(7), 61-63. https://doi.org/10.7202/1023501ar

Tous droits réservés @ Département des relations industrielles de l’Université Laval, 1949
Ce document est protégé par la loi sur le droit d'auteur. L’utilisation des services d'Érudit (y compris la reproduction) est assujettie à sa politique d'utilisation que vous pouvez consulter en ligne.

https://apropos.erudit.org/fr/usagers/politique-dutilisation/ 


\section{Bulletin des relations industrielles}

Volume 4, numéro 7

QUÉBEC

Mars 1949

Publié par le

Département des relations industrielles, Faculté des sciences sociales, Université Laval.

Georges-Henri Lévesque, o.p., doyen GérARD TrEmbLAy, directeur Gerard Dion, sous-directeur Jean Gagne, secrétaire

CharLes BÉlanger, administrateur

Le Bulletin paraît mensuellement de septembre à juin (dix numéros par année). Abonnement annuel: Canada: \$1.50; étranger: $\$ 2.00$. Vingt-cinq cents le numéro. Publication répertoriée dans le "Canadian Index".

\section{Adressez toute correspondance au secrétaire de rédaction Génard Dion} 2, rue de l'Université, Québec.

Bulletin des relations industrielles Volume 4, numero 7 Mars 1949

\section{Sommaire}

L'église catholique et le problème social

Marcel Clément.

Programme de formation professionrelle dans lindustrie de l'imprimerie de Montréal

Marcel Forget

Les congés annuels payés édictés par

décrets (Loi de la convention

collective)

Charles BÉlanger

Documentation relative ai Bill 5

Techniques des relations de travail Quatrième Congrès des relations

industrielles de Laval.

\section{Collaborateurs}

BÉl.ANgER, Charles, B.Sc.Soc., secrétaire du district de Québec de la Commission du salaire minimum et administrateur du Département des relations industrielles de la Faculté des sciences sociales de Laval.

Clément, Marcel, Licencié ès lettres, diplômé d'études supérieures en philosophie (Sorbonne), licencié en droit, diplômé d'études supérieures d'économie politique (Faculté de droit de Paris), professeur, Faculté des sciences sociales de Laval.

Fonget, Marcel, B.A., L.Ph., M.R.I., (Montréal), assistant-secrétaire de la Commission d'apprentissage des métiers de l'imprimerie de la cité de Montréal.

Autorisé comme envol postal de deuxlème classe, ministcre des posties, Ottoriva, Catiada.

\section{L'EGLISE CATHOLIQUE ET LE PROBLÈME SOCIAL}

\author{
Marcel Clément
}

On ne saurait exagérer la gravité douloureuse des problèmes sociaux qui se posent, depuis quelques semaines, dans un climat d'où la sérénité est absente. Ces problèmes sont graves, car c'est sur le fond même de la doctrine sociale de l'Eglise, pourtant si précise et si claire, que le désaccord s'exprime. Ils sont douloureux, parce qu'au delà de l'incroyable confusion des idées utilisées pour légitimer les passions, la souffrance des hommes augmente, la blessure faite au flanc d'une société, jusqu'ici heureuse et prospère si on la compare à tant d'autres, s'approfondit et même s'infecte. Ces jours de Carême sont propices à l'examen de conscience, car dans le domaine social comme dans le domaine familial, le désordre temporel résulte principalement des défaillances morales. Et au train où vont les choses, un tel retour sur soimême n'est plus seulement un acte de prudence. C'est l'unique voie de salut qui s'ouvre, si employeurs et employés veulent loyalement tenter de sortir de l'impasse diabolique où toute la vie sociale et économique est égarée avec eux.

L'Eglise a une attitude sans équivoque en face du problème social. Elle précise les droits et les devoirs des employeurs. Elle décrit les droits et les devoirs des employés. Enfin, elle situe dans sa juste perspective la fonction des pouvoirs publics. «Nous voulons parler des gouvernants, des maîtres et des riches, des ouvriers eux-mêmes dont le sort est ici en jeu. Ce que nous affirmons sans hésitation, c'est l'inanité de leur action en dehors de celle de l'Eglise. C'est l'Eglise, en effet, qui puise dans l'Evangile des doctrines capables soit de mettre fin au conflit, soit au moins de l'adoucir, en lui enlevant tout ce qu'il a d'âpreté et d'aigreur; l'Eglise, qui ne se contente pas d'éclairer l'esprit de ses enseignements, mais s'efforce de régler en conséquence la vie et les moeurs d'un chacun.» (Rerum novarum)

\section{I}

L'expérience quotidienne des relations du travail enseigne assez rapidement que le problème social, pour le patron, consiste essentiellement à vouloir améliorer l'ouvrier, et que pour l'employé, il consiste essentiellement à vouloir améliorer le patron. Très vite, d'ailleurs, ils désespèrent les uns et les 
autres d'y parvenir et considèrent que le seul instrument efficace c'est la contrainte. Dès lors, l'esprit de classe engendre la lutte des classes, et l'on voit un «patronat de combat » se dresser en face d'un «syndicalisme de revendication ». Le problème social n'est plus alors qu'une confrontation de force, où chacune des deux parties cherche des appuis auprès des pouvoirs publics. Quand les affaires en sont venues à ce point, utiliser les encycliques sociales, dans le jeu de la passion, c'est trahir leur esprit. Et c'est une faute qui peut coûter bien cher, car c'est tarir la source même de la grâce qui nous vient de ces textes.

* L'erreur capitale dans la question présente, c'est de croire que les deux classes sont ennemies nées l'une de l'autre, comme si la nature avait armé les riches et les pauvres pour qu'ils se combattent mutuellement dans un duel obstiné. » (Rerum novarum) Et participent à cette erreur, tous ceux qui, intéressés directement ou indirectement au problème, pensent qu'il $\mathrm{y} a$, dès ici-bas, le côté des 《bons» et le côté des «mauvais »; ceux qui ont la mentalité patronale décrivant, évoquant, classant, en un mot, méditant les fautes présumées de certains salariés ou de chefs qu'ils se sont donnés, ceux qui ont la mentalité ouvrière décrivant, évoquant, classant, et donc aussi méditant les injustices présumées des patrons. Et de même que quand deux époux ne vivent plus qu'en épiant les imperfections de l'autre, ils sont près de renoncer à vivre ensemble; de même, l'absence réciproque de charité rend inopérantes les techniques les mieux adaptées de la collaboration patronaleouvrière.

Telle est, pour le sociologue, l'évidence qui résume clairement la situation présente. Des deux côtés, on a décidé d'employer la force, qui jusqu'ici restait enveloppée de formules chrétiennes. Qu'on ne s'y trompe pas. Liexemple d'autres pays est instructif. La première bataille peut être gagnée par l'un ou par l'autre. De toute façon, la dernière consacrera nécessairement la défaite de la personne humaine dans des assauts où la passion, l'intérêt et l'orgueil auront rapidement défiguré tous les combattants.

\section{II}

S'il est vrai que la Providence tire le bien du mal, l'occasion est propice pour rechercher, au delà de la faute des hommes, (qu'un chrétien qui ne peut espérer être pardonné que dans la mesure où il accorde son propre pardon aurait tort de juger sans appel), la malfaisance du régime économique dans lequel nous vivons, et que Léon
XIII ne craignait pas de nommer \& le libéralisme amoral ». Il est beau de citer la lettre des encycliques et d'y rechercher des textes qui justifient passagèrement l'une ou l'autre attitude. Il serait plus conforme à l'esprit des encycliques sociales de préciser que le programme qu'elles tracent n'est pris au sérieux actuellement par d̀ peu près aucun groupe organisé.

Les encycliques sociales prévoient, dans l'entreprise, des réformes de structure: participation des salariés aux bénéfices, participation des ouvriers à la gestion, selon des formules prudentes et dans une loyauté réciproque. Où en est-on?

Les encycliques sociales prévoient, non un groupement général de défense des intérêts patronaux pour faire contrepoids aux unions ouvrières, mais la naissance, dans chaque industrie, dans chaque profession, de groupements patronaux, non pas destinés à la lutte des classes, mais à la prise en charge économique des problèmes de chaque profession. Où en est-on ?

Les encycliques prévoient la naissance, dans chaque industrie, dans chaque profession, de 《groupements corporatifs » réunissant organisations patronales et ouvrières en un corps unique, où s'élaborent en commun toutes les décisions relatives aux intérêts communs. Où en est-on ?

Les encycliques sociales prévoient le devoir de conscience pour chaque patron, de pratiquer, non seulement de justes salaires et des ajustements familiaux, mais de justes prix - qui ne sont pas les prix du profit maximum, mais d'un profit honnête. Où en est-on?

Les encycliques sociales prévoient le devoir de conscience, pour chaque salarié, de ne point léser son patron, de fournir tout le travail et la qualité du travail qu'il sait devoir fournir. Là encore, - et la question n'est pas moins opportune - où en est-on?

\section{III}

Tels sont les vrais problèmes. L'attitude de l'Eglise en face de la question sociale ne consiste pas dans une prise de position contre l'une ou contre l'autre des parties. Elle consiste à reprendre les coupables, à redresser la vérité, à indiquer et à inspirer, l'esprit d'amour fraternel qui, dans l'entreprise, dans la profession et dans la nation, peut seul permettre la mise en oeuvre des solutions techniques. Elle consiste à exhorter les patrons et les ouvriers, ceux qui s'en sentent capables, à prendre en mains la réalisation d'une économie corporative, unifiée dans un régime de col- 
laboration, par un esprit surnaturel d'amour fraternel, et ordonnée par la régulation morale des salaires et des prix. C'est quand tout va mal, quelquefois, que le Ciel inspire à l'homme l'énergie d'accomplir les actes que la routine quotidienne fait habituellement remettre au lendemain. C'est peut être l'occasion, pour quelquesuns de se faire les apôtres des encycliques sociales, réalisées en esprit et en vérité, et non plus utilisées par l'intérêt et la passion. «Les premiers apôtres, les apôtres immédiats des ouvriers seront des ouvriers, les apôtres du monde industriel et commerçant seront des industriels et des commerçants. » (Pie XI)

A quelqu'un qui demandait récemment:

«-Mais enfin! Il doit bien exister des techniques qui permettent de résoudre définitivement le problème social ! Ou bien est-ce que la science sociale n'est pas assez avancée »? - il fut répondu:

Pie XI a écrit: «Toutes les institutions destinées à favoriser la paix et l'entr'aide parmi les hommes, si bien conçues qu'elles paraissent, reçoivent leur solidité surtout du lien spirituel qui unit les membres entre eux. Quand ce lien fait défaut, une fréquente expérience montre que les meilleures formules restent sans résultats. » Les techniques sont indispensables. Mais que voulez-vous qu'on espère des techniques, là où les hommes se détestent ! L'infusion de la vie divine, d'une charité authentiquement surnaturelle dans les relations patronales-ouvrières pourra seule - mais combien y croient ? - permettre l'instauration du catholicisme social.

\title{
PROGRAMME DE FORMATION PROFESSIONNELLE DANS L'INDUSTRIE DE L'IMPRIMERIE DE MONTRÉAL
}

\author{
Marcel Forget
}

La Commission d'apprentissage de l'industrie de l'imprimerie de Montréal estime que la seule méthode de former ses apprentis de façon à les mettre en possession des secrets de leur métier est par un programme de formation professionnelle qui dispense les connaissances techniques et développe par le travail pratique les aptitudes naturelles propres au métier choisi. C'est pourquoi afin de donner à la formation professionnelle des assises solides et, par là, assurer à l'industrie de limprimerie une main-d'oeuvre compétente a-t-elle déterminé pour les apprentis soumis à sa juridiction des programmes d'études théoriques et de travaux pratiques, lesquels ont été approuvés par le Conseil de l'instruction publique.

\section{A-Formation théorique à l'Ecole des arts graphiques}

Comme la Loi de l'aide à l'apprentissage demande aux commissions d'apprentissage de collaborer avec les écoles d'enseignement spécialisé, la Commission d’apprentissage a recherché le moyen d'utiliser au mieux les services de l'Ecole des arts graphiques dans l'intérêt de l'industrie de l'imprımerie en général. Il lui a semblé que cette maison d'enseignement spécialisé constituait le milieu par excellence où les apprentis des première, deuxième et troisième années d'apprentissage pourraient $y$ recevoir, de jour, des cours de formation théorique - et ce, sur leur temps de travail et tout en étant rénumérés par leur employeur; où les apprentis des quatrième, cinquième et sixième années d'apprentissage pourraient y recevoir, le soir, un supplément de formation théorique et, par là, accroître leur valeur professionnelle et leur chance de promotion. C'est pourquoi elle a conclu, le 16 juillet 1948, avec le ministre du Bien-Etre social et de la Jeunesse de qui relève directement l'Ecole des arts graphiques, une entente à l'effet que ses apprentis aient l'opportunité de suivre à l'Ecole des arts graphiques, par mode de roulement et à raison d'une journée ou d'une soirée par semaine suivant le cas, le programme d'études théoriques qu'à leur intention elle a dressé en collaboration avec la direction de l'Ecole. des arts graphiques.

Dans l'économie de la règlementation, les cours de formation théorique du jour sont obligatoires et ont pour but de compléter et d'approfondir la formation pratique des apprentis en même temps que d'habituer les apprentis à continuellement faire appel à leurs facultés intellectuelles dans l'exercice de leur métier. Ils comprennent 